ehponline.org

\title{
Does Air Pollution Trigger \\ Infant Mortality in Western Europe? \\ A Case-Crossover Study
}

Hans Scheers, Samuel M. Mwalili, Christel Faes, Frans Fierens, Benoit Nemery, and Tim S. Nawrot

doi: 10.1289/ehp.1002913 (available at http://dx.doi.org/) Online 26 January 2011 


\title{
Does Air Pollution Trigger Infant Mortality in Western Europe? A Case-Crossover Study
}

\author{
Hans Scheers, ${ }^{1}$ Samuel M. Mwalili, ${ }^{2}$ Christel Faes, ${ }^{3}$ Frans Fierens, ${ }^{4}$ \\ Benoit Nemery, ${ }^{1}$ Tim S. Nawrot ${ }^{1,5}$
}

1. Department of Public Health, Occupational \& Environmental Medicine, K.U.Leuven, Leuven, Belgium.

2. Department of Biostatistics, K.U.Leuven, Leuven, Belgium, presently at Statistics and Actuarial Sciences, Jomo Kenyatta University of Agriculture and Technology, Nairobi, Kenya.

3. Center for Statistics, Hasselt University, Campus Diepenbeek, Diepenbeek, Belgium.

4. Belgian Interregional Environment Agency, Brussels, Belgium.

5. Centre for Environmental Sciences, Hasselt University, Campus Diepenbeek, Diepenbeek, Belgium.

\section{Corresponding author:}

Benoit Nemery

Department of Public Health, Occupational \& Environmental Medicine, K.U.Leuven Herestraat 49, O\&N 1, PB 706

3000 Leuven

Belgium

Tel. 003216347121 - Fax 003216347124

e-mail ben.nemery@med.kuleuven.be 
Running title: Air Pollution and Infant Mortality

Key words: acute effects, air pollution, case-crossover, epidemiology, infant mortality, particulate matter, SIDS

\section{Abbreviations used:}

AIC

AF

$\mathrm{Cl}$

EU

HRV

ICD-10

revision

NICU

OR

$\mathrm{PM}_{\mathrm{x}}$

$\leq \mathrm{x} \mu \mathrm{m}$

SIDS

WHO
Akaike's Information Criterion

Attributable Fraction

Confidence Interval

European Union

Heart Rate Variability

International Classification of Diseases, $10^{\text {th }}$

Neonatal Intensive Care Unit

Odds Ratio

Particulate Matter with an aerodynamic diameter

Sudden Infant Death Syndrome

World Health Organization

Contributions and acknowledgements: T. Nawrot, H. Scheers and B. Nemery designed the study. They had full access to all of the data in the study and take responsibility for the integrity of the data and the accuracy of the data analysis. $\mathrm{H}$. Scheers constructed the database and performed the analyses. S. Mwalili wrote a SAS macro to match the control days on temperature and C. Faes studied the shape of the association. F. Fierens interpolated the air pollution data. H. Scheers and T. Nawrot wrote the first draft of the manuscript. All authors interpreted the results and prepared the final version of the manuscript. 
The research on air pollution and health at K.U.Leuven is supported by the sustainable development program of BELSPO (Belgian Science Policy) and at UHasselt by a grant from the Flemish Scientific Fund (FWO, Krediet aan navorsers) and Bijzondere Onderzoekstoelagen. The sponsors of this study had no role in the study design, data management or preparation of the manuscript.

We thank Dr. A. Kongs from the Flemish Agency for Care and Health for the mortality data and the Flemish Environment Agency (VMM) for the air pollution data. No compensation was received for their contribution.

Competing financial interests declaration: We declare that we have no conflicts of interest. 


\section{ABSTRACT}

Background: Numerous studies show associations between fine particulate air pollutants $\left(\mathrm{PM}_{10}\right)$ and mortality in adults. We investigated short-term effects of elevated $\mathrm{PM}_{10}$ levels on infant mortality in Flanders, Belgium, and studied whether the European Union (EU) limit value protects infants from the air pollution trigger.

Methods: In a case-crossover analysis, we estimated the risk of dying from nontraumatic causes before the age of one year in relation with outdoor $\mathrm{PM}_{10}$ concentrations on the day of death. We matched control days on temperature in order to exclude confounding by variations in daily temperature.

Results: During the study period (1998-2006), $\mathrm{PM}_{10}$ concentration averaged $31.9 \mathrm{\mu g} / \mathrm{m}^{3}$ (SD 13.8). In the entire study population ( $\mathrm{N}=2382)$, the risk of death increased by $4 \%$ (95\% Cl: $0-8 \%, P=0.045$ ) for a $10 \mu \mathrm{g} / \mathrm{m}^{3}$ increase in daily mean $\mathrm{PM}_{10}$. However, this association was only significant for late neonates ( $2-4$ weeks of age, $N=372)$ in whom the risk of death increased by $11 \%(1-22 \%, \mathrm{P}=0.028)$ per $10 \mu \mathrm{g} / \mathrm{m}^{3}$ increase in $\mathrm{PM}_{10}$. In this age class, infants were $1.74(1.18-2.58, \mathrm{P}=0.006)$ times more likely to die on days with a mean $\mathrm{PM}_{10}$ above the EU limit value of $50 \mu \mathrm{g} / \mathrm{m}^{3}$ than on days below this cut-off. Conclusions: Even in an affluent region in Western Europe, where infant mortality is low, days with higher particulate air pollution are associated with an increased risk of infant mortality. Assuming causality, the current $E U$ limit value for $\mathrm{PM}_{10}$, which may be exceeded on 35 days per year, does not prevent $\mathrm{PM}_{10}$ from triggering mortality in late neonates. 


\section{INTRODUCTION}

In the past few decades, numerous studies have demonstrated that short-term exposure to elevated levels of air pollution has detrimental effects on human health. Most of these studies detected positive associations between particulate air pollution $\left(\mathrm{PM}_{10}\right.$ or $\mathrm{PM}_{2.5}$, i.e. particulate matter with an aerodynamic diameter less than 10 and $2.5 \mu \mathrm{m}$, respectively) and general mortality, or the triggering of acute cardiovascular events, especially in the elderly and people with pre-existing cardiovascular and respiratory conditions (Alfaro-Moreno et al. 2007; Pope 2000; Zanobetti and Schwartz 2005). In 1952, infant mortality doubled during the London smog (De Angelo and Black 2008; U.K.Ministry of Health 1954), but only recently has there been renewed concern about a possible link between exposure to air pollution and children's health. Children are considered as particularly susceptible to air pollution, since their lungs and immune system are immature during the first few years of life. Prenatal exposure to elevated levels of air pollution has been associated with early foetal loss, preterm delivery and lower birth weight (Bell et al. 2007; Schwartz 2004). Several studies investigated the association in infants (< one year of age) between particulate air pollution and all cause mortality, respiratory diseases or the Sudden Infant Death Syndrome (SIDS), yielding mixed results (Hajat et al. 2007; Kaiser et al. 2004; Lin et al. 2004; Romieu et al. 2004; Tsai et al. 2006; Woodruff et al. 2008; review in Glinianaia et al. 2004). The majority of these papers focused on urban areas in the United States or countries in transition, such as Brazil, Mexico and Taiwan, whereas the number of studies conducted in Western Europe is very limited.

The European Union (EU) set two limit values for $\mathrm{PM}_{10}$ concentrations: annual mean levels of $\mathrm{PM}_{10}$ must not exceed $40 \mu \mathrm{g} / \mathrm{m}^{3}$, and daily averages must not exceed $50 \mu \mathrm{g} / \mathrm{m}^{3}$ on more than 35 days per year. In contrast, the World Health Organization (WHO) 
argues that annual averages of $\mathrm{PM}_{10}$ levels should not be higher than $20 \mu \mathrm{g} / \mathrm{m}^{3}$ and that daily averages should not exceed $50 \mu \mathrm{g} / \mathrm{m}^{3}$ on more than three days per year (World Health Organization 2005).

Using a case-crossover analysis, we investigated whether there is an association between short-term elevations of $\mathrm{PM}_{10}$ levels and infant mortality over a recent 9-year period (1998-2006) in the region of Flanders, Belgium and we evaluated the effectiveness of the current EU limit values by exploring the possibility of a threshold value in the exposure - response curve. The densely populated Flemish region (> 6 million inhabitants on an area of $13500 \mathrm{~km}^{2}$, i.e. a population density of about 450 inhabitants $/ \mathrm{km}^{2}$ ) has very low rates of infant mortality by international standards (United Nations 2007), but also among the highest concentrations of $\mathrm{PM}_{10}$ in Europe, with frequent exceedings of the prevailing EU limit values (Beelen et al. 2009; Nawrot et al. 2007). Main sources of $\mathrm{PM}_{10}$ emission are traffic, industry and agriculture.

In our analyses, we took into account the effect of socio-economic status (SES), since SES has been shown to be a possible modifier of the association between air pollution and health (Carbajal-Arroyo et al. 2010).

\section{MATERIALS AND METHODS}

\section{Collection of data}

Mortality data. We obtained data of daily infant mortality in Flanders during the period 1998-2006 from the Flemish Agency for Care and Health. These data were anonymous, but the following information was provided: date of death; postal code of municipality of residence; official cause of death, according to the International Classification of Diseases, 10th revision (ICD-10); maturity at birth (a binary variable: mature or premature, i.e. below 37 weeks of gestation); and age at death categorized (according 
to the WHO classification) as: early neonatal ( $\leq 7$ days of age), late neonatal ( 8 to 28 days of age) or postneonatal (29 to 365 days of age).

Air pollution data. In Belgium, $\mathrm{PM}_{10}$ and several other indicators of ambient air quality are continuously measured by a dense network of automatic monitoring sites (see website: www.irceline.be). 19 of these measurement stations have been in use in the Region of Flanders from 1998 on, and they are situated $25 \mathrm{~km}$ apart from each other on average. Using a land use regression model (Janssen et al. 2008), we calculated the daily exposure level of $\mathrm{PM}_{10}$ at the municipality level for each mortality case. This model provides interpolated $\mathrm{PM}_{10}$ values from the Belgian telemetric air quality network in 4 by $4 \mathrm{~km}$ grids. The interpolation is based on a detrended Kriging interpolation model that uses landcover data obtained from satellite images (Corine landcover dataset) (Janssen et al. 2008).

Temperature data. Temperature is a known confounder of the association between air pollution and mortality (Hajat et al. 2002; Huynen et al. 2001; Katsouyanni et al. 1997; Nawrot et al. 2007). We obtained daily average temperatures from the Belgian Royal Meteorological Institute. The Region of Flanders is very uniform for temperature, since both altitudinal and latitudinal gradients are extremely small: elevations range from 0 to $200 \mathrm{~m}$ above sea level and the distance between the northernmost and southernmost part is only $100 \mathrm{~km}$. The region is not larger than the state of Connecticut (United States). Therefore, we used temperature data from the central and representative station in Uccle (Brussels).

Socioeconomic status. We created three classes of SES on the municipality level, based on characteristics including salary level, economic activity, degree of unemployment and housing grade equipment. (Dexia Bank NV 2007). 


\section{Analytical strategy}

Case-crossover design. We investigated the association between air pollution and infant mortality using a case-crossover design, a technique developed by Maclure (1991) which combines features of the crossover design and the matched case-control design. Similar to a crossover study, each subject serves as his own control and, as in matched case-control studies, the inference is based on a comparison of exposure distribution rather than the risk of disease (Jaakkola 2003). The case-crossover design is now widely used for analyzing short-term health effects of air pollution (Carracedo-Martinez et al. 2010).

Selection of hazard period and control days (Figure 1). We defined the hazard period, which is the brief time period when a subject is at risk, as the day of death (event day). We selected control days based on three criteria. First, we took control days from the same calendar month and year as the event days, both before and after the event. We chose this bidirectional time-stratified design above other selection strategies in order to avoid issues of bias, as explained by Janes et al. (2005) and Mittleman (2005). Second, control days and event days had to be at least three days apart from each other in order to avoid short-term autocorrelation (Levy et al. 2001). This implies a five-day exclusion period around the event day. Third, since temperature is a known confounder of the association between air pollution and health (Hajat et al. 2002; Huynen et al. 2001; Katsouyanni et al. 1997; Nawrot et al. 2007), we only selected control days having a daily average temperature within $2^{\circ} \mathrm{C}$ of that on the event day. Based on this strategy, the number of control days per event ranged from zero to a maximum of 25 , depending on the temperature criterion. On average, each case had 8.6 control days. 76 cases (3.2\%) had no control days and were, by consequence, not included in the analyses. 
Shape of the association. In order to investigate whether there might be a threshold level in the exposure - response relationship or a plateau at higher concentrations, we studied the shape of the association between $\mathrm{PM}_{10}$ and risk of death by the use of fractional polynomials. Although linear and quadratic polynomials are commonly used, they are often inadequate to describe the shape of the association. Fractional polynomials are an alternative to classical polynomials, but still fall within the realm of (generalized) linear methods. They extend the classical linear and quadratic models by allowing any power from a predefined set of values typically chosen from the set $(-2,-1,-1 / 5,0,1 / 5,1,2,3)$ (Royston and Altman 1994). From this family of models, the best functional form is chosen using Akaike's Information Criterion (AIC). A particular feature of the fractional polynomials is that they provide a wide class of functional forms, with only a small number of terms. Moreover, the conventional linear and quadratic polynomials are included as a subset of this extended family. Based on the best fitting model, we calculated odds ratios (ORs) for mortality in association with a $10 \mu \mathrm{g} / \mathrm{m}^{3}$ increase in $\mathrm{PM}_{10}$.

Additional analyses. To detect a possible short-term delay in the effects of exposure to $\mathrm{PM}_{10}$, we performed five additional case-crossover analyses with different lag structures. In these analyses, we defined the hazard period as 1, 2 or 3 days before the day of death (lag day 1, lag day 2 and lag day 3, respectively) or as the moving average exposure on two (event day and lag day 1 ) or three (event day and lag days 1 and 2 ) consecutive days. We also performed a sensitivity analysis using an alternative selection strategy, with control days being matched on day of the week instead of daily temperature, thus also including the 76 cases that had no temperature-matched control day.

We conducted stratified analyses by age class, by maturity, by SES, and by cause of death, categorized as cardiorespiratory diseases (ICD-10 I00-J99), SIDS (ICD-10 R95), 
perinatal circumstances (ICD-10 P00-P96), congenital and chromosomal abnormalities (Q00 - Q99) or other. Infants who died from external causes (e.g. accidents, ICD-10 V00-Y98, $\mathrm{N}=73$ ) were excluded from all analyses.

Finally, we transformed the exposure value into a binary variable (i.e. below or above the EU limit value of $50 \mu \mathrm{g} / \mathrm{m}^{3}$ ) and calculated the ORs for dying on days above $50 \mu \mathrm{g} / \mathrm{m}^{3}$ compared to days with $\mathrm{PM}_{10}$ levels below that value.

Statistical analyses. Database management and statistical analyses were performed in SAS software, version 9.1. (SAS Institute, Cary, NC, USA). We used conditional logistic regression to evaluate the case-crossover data and to estimate the odds of all-cause and cause-specific infant mortality by exposure to $\mathrm{PM}_{10}$. Results are presented as ORs with $95 \%$ confidence intervals $(\mathrm{Cl})$ per $10 \mu \mathrm{g} / \mathrm{m}^{3}$ increment in $\mathrm{PM}_{10}$ concentration or as the OR for days above the EU limit value of $50 \mu \mathrm{g} / \mathrm{m}^{3}$ against days below that value. We calculated the attributable fraction (AF) as in (Steenland and Armstrong 2006). All tests were two-sided with $\alpha=0.05$.

\section{RESULTS}

\section{Descriptive Data}

Of the 2455 infants who died in Flanders during the study period (1998-2006) (yielding a mortality of 4.67 per 1000 live births), 2382 died from non-traumatic causes, and 1284 infants (54\%) had been born before 37 weeks of gestation. Figures on age at death and causes of death are provided in Table 1. During the study period, $\mathrm{PM}_{10}$ concentration averaged $31.9 \mu \mathrm{g} / \mathrm{m}^{3}$ (SD 13.8) (Figure $2 \mathrm{~A}-\mathrm{B}$ ) and there were 321 days (an average of 35.7 days per year) with a mean daily concentration exceeding $50 \mu \mathrm{g} / \mathrm{m}^{3}$ (populationweighted daily average for the whole region). For cases $(\mathrm{N}=2382)$, the average exposure was $32.6 \mu \mathrm{g} / \mathrm{m}^{3}(95 \% \mathrm{Cl} 15.1-59.9)$ and on the selected control days $(\mathrm{N}=20448) \mathrm{PM}_{10}$ averaged $30.7 \mu \mathrm{g} / \mathrm{m}^{3}$ (95\% $\left.\mathrm{Cl} 14.8-56.5\right)$. Interpolated daily average $\mathrm{PM}_{10}$ 
concentrations were strongly correlated among the 308 municipalities in the study area. Correlations ranged from 0.87 to 1.00 and the strongest correlations were found among neighbouring municipalities.

\section{Case-crossover analysis}

For the whole group, we found a $4 \%$ increase $(95 \% \mathrm{Cl}: 0-8 \%, P=0.045)$ in the risk of death for each $10 \mu \mathrm{g} / \mathrm{m}^{3}$ increase in the concentration of $\mathrm{PM}_{10}$ on the event day (lag day 0) (Table 2). In the sensitivity analyses with up to three lag days or moving average concentrations, mortality tended to be positively associated with $\mathrm{PM}_{10}$ as well, but these associations were not significant (data not shown). Estimates from analyses with control days matched on day of the week were comparable to those with control days matched on temperature (data not shown). Therefore, we further report only results for exposure to $\mathrm{PM}_{10}$ on the event day compared with temperature-matched control days.

Stratification by age class revealed stronger associations with deaths between two to four weeks of age (late neonates) than with deaths during other time periods.

Specifically, a $10 \mu \mathrm{g} / \mathrm{m}^{3}$ increase in mean daily $\mathrm{PM}_{10}$ on the event day was associated with an $11 \%$ increase $(1-22 \%, P=0.028)$ in the risk of late neonatal death. In contrast we found no evidence of effects of $\mathrm{PM}_{10}$ on early neonatal or postneonal mortality. Stratified analyses revealed no significant differences in associations between $\mathrm{PM}_{10}$ and daily mortality among preterm versus term births (P's for interaction $\geq 0.09$, Table 2 ), although odds ratios were always higher for the latter group. We further analyzed the relation between air pollution and mortality according to cause of death (Table 3). In the total group we found no significant associations between $\mathrm{PM}_{10}$ and mortality from cardiorespiratory diseases or SIDS. In contrast, in the total group, $\mathrm{PM}_{10}$ and mortality were significantly associated in cases where the cause of death was 
determined as 'perinatal circumstances'. For late neonatal deaths, the associations were mainly driven by the group with 'congenital and chromosomal abnormalities' (Table 3). Analyses in which the EU limit value of $50 \mu \mathrm{g} / \mathrm{m}^{3}$ was taken as a cut-off point revealed a non-significant OR for the whole group, but a highly significant result for late neonatal mortality with an OR for dying on days with $\mathrm{PM}_{10}$ above $50 \mu \mathrm{g} / \mathrm{m}^{3}$ of $1.74(1.18-2.58 ; P=$ 0.006), compared to days below the cut-off value (Table 2). The corresponding AF was $43 \%(15-61 \%)$. When we stratified the analysis with the EU limit value by cause of death, the highest OR (for 'congenital and chromosomal abnormalities') was just not significant. For other causes of death, results were not significant either. For late neonatal deaths however, stratification by cause of death revealed a highly significant result for 'congenital and chromosomal abnormalities' ( $P=0.009)$ (Table 3). In all settings, subanalyses of congenital malformations of the circulatory or respiratory system (Q20-Q28 and Q30-Q34, respectively) revealed similar ORs as in the whole group of congenital and chromosomal abnormalities (Q00-Q99), but due to a smaller sample size, these results did not reach statistical significance.

Analyses stratified by SES (low, medium or high SES municipality) were consistent with those estimated for the population as a whole, though ORs within SES categories were non-significant. ORs did not differ substantially among SES categories, as indicated by the non-significant interaction terms in all analyses (results not shown). In the group of late neonates, fractional polynomial analysis revealed that a linear model adequately describes the association between infant mortality and air pollution, with no evidence for a threshold or plateau (Likelihood Ratio test for a linear model versus a null model, $P=0.030$ ) (Figure 3). More complex fractional polynomials did not significantly improve the fit of the model, according to AIC. 


\section{DISCUSSION}

The key finding of our study was that particulate air pollution, expressed as $\mathrm{PM}_{10}$, is associated with late neonatal mortality, thus suggesting that airborne particles act as a rapid trigger of infant death. On days with average $\mathrm{PM}_{10}$ levels exceeding the $\mathrm{EU}$ limit value of $50 \mathrm{\mu g} / \mathrm{m}^{3}$ - which is allowed to be exceeded on 35 days per year - the odds for late neonatal mortality was 1.74 times higher than on days below that value. Assuming causality, these results imply that on days above the EU limit value of $50 \mu \mathrm{g} / \mathrm{m}^{3}, 43 \%$ (the AF) of late neonatal mortality could be triggered by an acute increase in fine particulate air pollution levels on the same day. The shape of the association between the risk of late neonatal mortality and $\mathrm{PM}_{10}$ (Figure 3) gives no evidence for a threshold, thus suggesting the risk exists even below $50 \mu \mathrm{g} / \mathrm{m}^{3}$. Analyses of lagged exposures suggested that exposure on the event day was more important than exposure during the 3 days preceding the event.

Most publications on infant mortality and PM air pollution have used a time-series approach. The case-crossover design represents a relatively novel approach to study acute health effects. It was developed in the early 1990s by Maclure (1991) to study effects of brief exposures on the change in risk of acute and discrete events, such as myocardial infarction. Recently, the case-crossover design has been applied to assess effects of short-term changes in exposure to air pollution (e.g. Romieu et al. 2004; Son et al. 2008; Tsai et al. 2006; Yang et al. 2006; Zanobetti and Schwartz 2005; review in Carracedo-Martinez et al. 2010). The major power of the approach is the ability to control for confounding. In the case-crossover design, all the study subjects have experienced the event. The hazard period is defined as the average time period that is relevant for the acute event, and this period is compared with control times. Thus, subjects serve as their own controls at an individual level. In contrast, the traditional time-series studies cannot 
control for varying individual characteristics since the unit of observation consists of daily counts of the event rather than of individuals. By matching for outdoor temperature, we excluded temperature as a potential confounder in our models and, since control days were close to event days, we controlled for seasonal effects as well (Bateson and Schwartz 1999, 2001; Maclure and Mittleman 2000). The time-stratified design for the selection of control days, as applied in our study, has been shown to be the best selection method to avoid statistical bias (Janes et al. 2005; Mittleman 2005).

So far, only five case-crossover studies on infant mortality and air pollution have been published. These were conducted in the cities of Seoul (South Korea) (Son et al. 2008), Kaohsiung (Taiwan) (Tsai et al. 2006), Taipei (Taiwan) (Yang et al. 2006), Ciudad Juárez (Mexico) (Romieu et al. 2004), and Mexico City (Mexico) (Carbajal-Arroyo et al. 2010). Apart from the latter, they found no short-term association between postneonatal mortality and air pollution (OR's were 1.00 or 1.01 for an increase of $10 \mu \mathrm{g} / \mathrm{m}^{3}$ ). In contrast to these studies, which exclusively dealt with postneonatal mortality (> one month of age), we also included neonates in the analysis. In our study, we observed no evidence of an association between $\mathrm{PM}_{10}$ and postneonatal mortality either, but we estimated a significant positive association between a $10 \mu \mathrm{g} / \mathrm{m}^{3}$ increase in $\mathrm{PM}_{10}$ and mortality on the same day for all age classes combined that was almost entirely due to an association between $\mathrm{PM}_{10}$ and mortality during the late neonatal period (two to four weeks after birth). In both studies performed in Mexico (Carbajal-Arroyo et al. 2010; Romieu et al. 2004), the risk of death was significantly higher in infants from low and/or medium SES areas than in those from high SES areas. We found no difference in ORs among municipalities classified according to SES. Due to privacy restrictions we were not able to classify SES on an individual level, but for the present, we conclude that SES does not modify the association between PM exposure and infant death in the study region. We found no indications for a role of $\mathrm{PM}_{10}$ in infants who died from 
cardiorespiratory complications or SIDS. Earlier studies on the association between exposure to PM and SIDS yielded mixed results (Glinianaia et al. 2004; Tong and Colditz 2004), though our results for cardiorespiratory deaths may be unreliable due to the very small sample size. In the present study we estimated the highest ORs for deaths attributed to congenital malformations and perinatal circumstances, but only the latter proved to be significant for the whole study population and only the former for deaths among late neonates.

We did not find clear evidence of differences between term and preterm births, and neither could we detect a significant association between air pollution and early neonatal mortality during the first week of life. Reasons for this might be that the most susceptible children die during the first week of life due to conditions that do not need to be triggered by air pollution, or that the measured outdoor air pollution does not reflect actual exposure during the first week of life (or during the first month for premature infants), because most of these newborns probably would have remained in the hospital during this time. However, we had no access to data on the duration of hospitalization after birth to verify this hypothesis.

In this context, a limitation of our study is the use of outdoor measurements of air pollution with interpolations at the municipality level in order to estimate partly indoor personal exposures. However, recent studies (Janssen et al. 2005; Williams et al. 2000) comparing personal with ambient exposure have reported good correlations between day to day changes in central measurement stations of particulates and personal exposure. In addition, we found very high correlations (ranging from 0.87 to 1 ) among municipalities for the interpolated $\mathrm{PM}_{10}$ levels. In other words, spatial variability in $\mathrm{PM}_{10}$ (which is rather low in our small study area) appeared to be less important than temporal variability, which is driven largely by weather conditions. During stable meteorological conditions with low wind speeds, and the presence of a temperature inversion, locally- 
produced pollution accumulates in the lower parts of the atmosphere, which results in a cloud of dust inhaled by humans.

In their comprehensive review, Pope and Dockery (2006) discuss several plausible biological pathways for the relationship between exposure to PM and health. Evidence for these pathways was mainly derived from observations on adults or experiments on animals, but at least some of the proposed mechanisms, such as pulmonary or systemic inflammation and modulated immunity, are likely to explain adverse health effects in infants as well, since their lungs, heart and immune system are immature and fragile. In particular, there is growing evidence that ambient air pollution is associated with decreased heart rate variability (HRV) in adults (Pope and Dockery 2006) and reduction in $\mathrm{HRV}$ is a plausible biological mechanism in infant deaths as well (Patzak 1999). We did not find significant associations between $\mathrm{PM}_{10}$ and cardiorespiratory diseases as the official cause of death, but the number of children in this group was very low, which in turn might be the consequence of misclassification on death certificates (subtle mechanisms as systemic inflammation or HRV are presumably more easily overlooked than perinatal or congenital abnormalities, see Nembhard et al. (2008) and references therein for examples of misclassification of cardiovascular diseases). Hence, there is clearly a need for further research in order to understand the underlying mechanisms of the observed associations between air pollution and mortality in infants, as well as a better differentiation between acute and chronic effects of air pollution in this segment of the population.

\section{CONCLUSIONS}

Our study shows that air pollution standards have to be taken more seriously. We estimated that $43 \%$ of mortality during the late neonatal period may be triggered by peaks of $\mathrm{PM}_{10}$ above $50 \mu \mathrm{g} / \mathrm{m}^{3}$. We do not claim that air pollution was the major, let 
alone the only cause of death in these infants, but our data suggest that air pollution may precipitate death in infants with preexisting conditions. A trigger is not necessarily the primary cause of death, but it may increase the risk of death in susceptible infants, such as infants with perinatal complications or other pre-existing conditions.

European regulation, which currently uses standards that are considerably higher than those of the WHO (World Health Organization 2005), stipulates that we may have a maximum of 35 days with a mean $\mathrm{PM}_{10}$ above $50 \mu \mathrm{g} / \mathrm{m}^{3}$ per year [comparable to the United States Environmental Protection Agency standard for $\mathrm{PM}_{2.5}$ of $35 \mu \mathrm{g} / \mathrm{m}^{3}(\sim 46$ $\left.\left.\mu \mathrm{g} / \mathrm{m}^{3} \mathrm{PM}_{10}\right)\right]$. In Belgium, this standard is barely met at present and although minor improvements due to emission reduction measures are expected, the limit value of 50 $\mu \mathrm{g} / \mathrm{m}^{3}$ will continue to be exceeded frequently in the coming decade. The same is largely true for other European regions including Northern France, the Southern part of the Netherlands, the German Ruhr area and the Po valley in Italy. The argument that it is difficult to meet standards in densely populated areas ignores the fact that the importance of a factor with respect to public health increases in proportion to the number of people that are exposed to it. 


\section{Reference List}

Alfaro-Moreno E, Nawrot TS, Nemmar A, Nemery B. 2007. Particulate matter in the environment: pulmonary and cardiovascular effects. Curr Opin Pulm Med 13:98-106.

Bateson TF, Schwartz J. 1999. Control for seasonal variation and time trend in case-crossover studies of acute effects of environmental exposures. Epidemiology 10:539-544.

Bateson TF, Schwartz J. 2001. Selection bias and confounding in case-crossover analyses of environmental time-series data. Epidemiology 12:654-661.

Beelen R, Hoek G, Pebesma E, Vienneau D, de Hoogh K, Briggs DJ. 2009. Mapping of background air pollution at a fine spatial scale across the European Union. Sci Total Environ 407:1852-1867.

Bell ML, Ebisu K, Belanger K. 2007. Ambient air pollution and low birth weight in Connecticut and Massachusetts. Environ Health Perspect 115:1118-1124.

Carbajal-Arroyo L, Miranda-Soberanis V, Medina-Ramon M, Rojas-Bracho L, Tzintzun G, SolisGutierrez P et al. 2010. Effect of PM10 and O3 on infant mortality among residents in the Mexico City Metropolitan Area: a case-crossover analysis, 1997-2005. J Epidemiol Community Health. Published Online First: 18 August 2010. doi:10.1136/jech.2009.101212

Carracedo-Martinez E, Taracido M, Tobias A, Saez M, Figueiras A. 2010. Case-crossover analysis of air pollution health effects: a systematic review of methodology and application. Environ Health Perspect 118:1173-1182.

De Angelo L, Black B. 2008. London smog disaster, England. In: Encylopedia of Earth (Cleveland CJ, ed.). Washington D.C.:National Council for Science and the Environment.

Dexia Bank NV. 2007. Sociaaleconomische typologie van de gemeenten (Socioeconomic typology of the municipalities). http://www.dexia.be/NI/smallsites/research/PublicFinance/typo/ [accessed 19 january 2011].

Glinianaia SV, Rankin J, Bell R, Pless-Mulloli T, Howel D. 2004. Does particulate air pollution contribute to infant death? A systematic review. Environ Health Perspect 112:1365-1371. 
Hajat S, Armstrong B, Wilkinson P, Busby A, Dolk H. 2007. Outdoor air pollution and infant mortality: analysis of daily time-series data in 10 English cities. J Epidemiol Community Health 61:719-722.

Hajat S, Kovats RS, Atkinson RW, Haines A. 2002. Impact of hot temperatures on death in London: a time series approach. J Epidemiol Community Health 56:367-372.

Huynen MM, Martens P, Schram D, Weijenberg MP, Kunst AE. 2001. The impact of heat waves and cold spells on mortality rates in the Dutch population. Environ Health Perspect 109:463-470.

Jaakkola JJ. 2003. Case-crossover design in air pollution epidemiology. Eur Respir J Suppl 40:81s-85s.

Janes H, Sheppard L, Lumley T. 2005. Overlap bias in the case-crossover design, with application to air pollution exposures. Stat Med 24:285-300.

Janssen NA, Lanki T, Hoek G, Vallius M, de Hartog JJ, Van Grieken R, et al. 2005. Associations between ambient, personal, and indoor exposure to fine particulate matter constituents in Dutch and Finnish panels of cardiovascular patients. Occup Environ Med 62:868-877.

Janssen S, Dumont G, Fierens F, Mensink C. 2008. Spatial interpolation of air pollution measurements using CORINE land cover data. Atmospheric Environment 42:4884-4903.

Kaiser R, Romieu I, Medina S, Schwartz J, Krzyzanowski M, Kunzli N. 2004. Air pollution attributable postneonatal infant mortality in U.S. metropolitan areas: a risk assessment study. Environ Health 3:4.

Katsouyanni K, Touloumi G, Spix C, Schwartz J, Balducci F, Medina S et al. 1997. Short-term effects of ambient sulphur dioxide and particulate matter on mortality in 12 European cities: results from time series data from the APHEA project. Air Pollution and Health: a European Approach. BMJ 314:1658-1663.

Levy D, Lumley T, Sheppard L, Kaufman J, Checkoway H. 2001. Referent selection in casecrossover analyses of acute health effects of air pollution. Epidemiology 12:186-192.

Lin CA, Pereira LA, Nishioka DC, Conceicao GM, Braga AL, Saldiva PH. 2004. Air pollution and neonatal deaths in Sao Paulo, Brazil. Braz J Med Biol Res 37:765-770.

Maclure M. 1991. The case-crossover design: a method for studying transient effects on the risk of acute events. Am J Epidemiol 133:144-153. 
Maclure M, Mittleman MA. 2000. Should we use a case-crossover design? Annu Rev Public Health 21:193-221.

Mittleman MA. 2005. Optimal referent selection strategies in case-crossover studies: a settled issue. Epidemiology 16:715-716.

Nawrot TS, Torfs R, Fierens F, De Henauw S, Hoet PH, Van Kersschaever G et al. 2007. Stronger associations between daily mortality and fine particulate air pollution in summer than in winter: evidence from a heavily polluted region in western Europe. J Epidemiol Community Health 61:146-149.

Nembhard WN, Pathak EB, Schocken DD (2008) Racial/ethnic disparities in mortality related to congenital heart defects among children and adults in the United States. Ethn Dis 18:442-449.

Patzak A. 1999. Short-term rhythms of the cardiorespiratory system and their significance in neonatology. Chronobiol Int 16:249-268.

Pope CAI. 2000. Epidemiology of fine particulate air pollution and human health: biologic mechanisms and who's at risk? Environ Health Perspect 108 Suppl 4:713-723.

Pope CAI, Dockery DW. 2006. Health effects of fine particulate air pollution: lines that connect. J Air Waste Manag Assoc 56:709-742.

Romieu I, Ramirez-Aguilar M, Moreno-Macias H, Barraza-Villarreal A, Miller P, HernandezCadena $L$ et al. 2004. Infant mortality and air pollution: modifying effect by social class. J Occup Environ Med 46:1210-1216.

Royston P, Altman DG. 1994. Regression using fractional polynomials of continuous covariates: Parsimonious parametric modelling. Appl Statist 43:429-467.

Schwartz J. 2004. Air pollution and children's health. Pediatrics 113:1037-1043.

Son JY, Cho YS, Lee JT. 2008. Effects of air pollution on postneonatal infant mortality among firstborn infants in Seoul, Korea: case-crossover and time-series analyses. Arch Environ Occup Health 63:108-113.

Steenland K, Armstrong B. 2006. An overview of methods for calculating the burden of disease due to specific risk factors. Epidemiology 17:512-519.

Tong S, Colditz P. 2004. Air pollution and sudden infant death syndrome: a literature review. Paediatr Perinat Epidemiol 18:327-335. 
Tsai SS, Chen CC, Hsieh HJ, Chang CC, Yang CY. 2006. Air pollution and postneonatal mortality in a tropical city: Kaohsiung, Taiwan. Inhal Toxicol 18:185-189.

U.K.Ministry of Health 1954. Mortality and Morbidity during the London Fog of December 1952. Reports on Public Health and Medical Subjects No. 95. London, United Kingdom, Ministry of Health.

United Nations. 2007. World Population Prospects: The 2006 Revision, Highlights, Working Paper No. ESA/P/WP. Department of Economic and Social Affairs. New York, United Nations.

Williams R, Suggs J, Zweidinger R, Evans G, Creason J, Kwok R et al. 2000. The 1998 Baltimore Particulate Matter Epidemiology-Exposure Study: part 1. Comparison of ambient, residential outdoor, indoor and apartment particulate matter monitoring. J Expo Anal Environ Epidemiol 10:518-532.

Woodruff TJ, Darrow LA, Parker JD. 2008. Air pollution and postneonatal infant mortality in the United States, 1999-2002. Environ Health Perspect 116:110-115.

World Health Organization. 2005. WHO air quality guidelines for particulate matter, ozone, nitrogen dioxide and sulphur dioxide: global update http://www.who.int/phe/health_topics/outdoorair_agg/en/ [accessed 19 January 2011].

Yang CY, Hsieh HJ, Tsai SS, Wu TN, Chiu HF. 2006. Correlation between air pollution and postneonatal mortality in a subtropical city: Taipei, Taiwan. J Toxicol Environ Health A 69:20332040.

Zanobetti A, Schwartz J. 2005. The effect of particulate air pollution on emergency admissions for myocardial infarction: a multicity case-crossover analysis. Environ Health Perspect 113:978982. 


\section{Tables}

\begin{tabular}{|c|c|c|c|c|}
\hline $\begin{array}{l}\text { Cause of death } \\
\text { (ICD-10 code) }\end{array}$ & $\begin{array}{l}\text { Early neonatal } \\
\text { ( } \leq 7 \text { days of } \\
\text { age) }\end{array}$ & $\begin{array}{c}\text { Late neonatal } \\
\text { (8 to } 28 \text { days of } \\
\text { age) }\end{array}$ & $\begin{array}{c}\text { Postneonatal } \\
\text { (29 to } 365 \text { days } \\
\text { of age) }\end{array}$ & Total \\
\hline $\begin{array}{l}\text { Cardiorespiratory diseases } \\
(\mathrm{I00}-\mathrm{J} 99)\end{array}$ & 3 & 3 & 44 & 50 \\
\hline $\begin{array}{l}\text { Perinatal circumstances } \\
\text { (P00 - P96) }\end{array}$ & 771 & 197 & 126 & 1094 \\
\hline $\begin{array}{l}\text { Congenital and chromosomal } \\
\text { abnormalities (Q00 - Q99) }\end{array}$ & 398 & 140 & 205 & 743 \\
\hline SIDS (R95) & 0 & 0 & 285 & 285 \\
\hline Others & 24 & 22 & 164 & 210 \\
\hline Total & 1196 & 372 & 814 & 2382 \\
\hline
\end{tabular}




\begin{tabular}{|c|c|c|c|c|}
\hline Age category & $\begin{array}{c}\text { All } \\
(\mathrm{N}=2382)\end{array}$ & $\begin{array}{l}\text { Preterm } \\
(\mathrm{N}=1284)\end{array}$ & $\begin{array}{c}\text { Term } \\
(\mathrm{N}=1086)\end{array}$ & $P$ interaction $^{\mathrm{a}}$ \\
\hline \multicolumn{5}{|c|}{ OR for $10 \mu \mathrm{g} / \mathrm{m}^{3}$ increase in $\mathrm{PM}_{10}$ on event day } \\
\hline All & $1.04(1.00 \text { to } 1.08)^{*}$ & 1.03 (0.98 to 1.08$)$ & 1.05 (0.99 to 1.11$)$ & 0.62 \\
\hline Early neonatal & 1.04 (0.99 to 1.10$)$ & 1.03 (0.96 to 1.10$)$ & 1.07 (0.97 to 1.19$)$ & 0.49 \\
\hline Late neonatal & $1.11(1.01 \text { to } 1.22)^{*}$ & 1.10 (0.97 to 1.24$)$ & 1.13 (0.98 to 1.31$)$ & 0.77 \\
\hline Post neonatal & 1.01 (0.95 to 1.07$)$ & 0.99 (0.88 to 1.10$)$ & $1.02(0.94$ to 1.10$)$ & 0.67 \\
\hline \multicolumn{5}{|c|}{ OR for days above $50 \mu \mathrm{g} / \mathrm{m}^{3}$ vs. days below $50 \mu \mathrm{g} / \mathrm{m}^{3}$ b } \\
\hline All & $1.10(0.94$ to 1.29$)$ & $0.96(0.76$ to 1.20$)$ & $1.27(1.01 \text { to } 1.61)^{*}$ & 0.09 \\
\hline Early neonatal & 0.99 (0.78 to 1.24$)$ & 0.92 (0.69 to 1.22$)$ & $1.14(0.75$ to 1.74$)$ & 0.40 \\
\hline Late neonatal & $1.74(1.18 \text { to } 2.58)^{* *}$ & $1.47(0.87$ to 2.48$)$ & $2.09(1.15 \text { to } 3.79)^{*}$ & 0.38 \\
\hline Post neonatal & 1.04 (0.79 to 1.37$)$ & 0.74 (0.43 to 1.27$)$ & $1.18(0.86$ to 1.63$)$ & 0.14 \\
\hline
\end{tabular}

Data are ORs with 95\% Cl. " $P \leq 0.05$ and ${ }^{* *} P \leq 0.01$.

${ }^{a}$ Interaction between exposure and maturity at birth, with preterm birth defined as born before 37 weeks of gestation

${ }^{\mathrm{b}}$ Based on EU limit value. 


\begin{tabular}{|c|c|c|c|c|}
\hline \multirow[t]{2}{*}{ Cause of death (ICD-10) } & All & Early neonatal & Late neonatal & Post-neonatal \\
\hline & \multicolumn{4}{|c|}{ OR for $10 \mu \mathrm{g} / \mathrm{m}^{3}$ increase in $\mathrm{PM}_{10}$ on event day } \\
\hline Total & $1.04(1.00 \text { to } 1.08)^{*}$ & $1.04(0.99$ to 1.10$)$ & $1.11(1.01 \text { to } 1.22)^{*}$ & $1.01(0.95$ to 1.07$)$ \\
\hline Cardiorespiratory diseases & $0.98(0.78$ to 1.25$)$ & na & na & $0.98(0.76$ to 1.26$)$ \\
\hline Perinatal circumstances & $1.06(1.00 \text { to } 1.12)^{*}$ & $1.06(1.00 \text { to } 1.14)^{*}$ & 1.09 (0.95 to 1.25$)$ & $1.01(0.86$ to 1.19$)$ \\
\hline $\begin{array}{l}\text { Congenital and } \\
\text { chromosomal abnormalities }\end{array}$ & 1.04 (0.97 to 1.12$)$ & $1.00(0.91$ to 1.11$)$ & $1.16(1.00 \text { to } 1.35)^{*}$ & $1.04(0.90$ to 1.20$)$ \\
\hline \multirow[t]{2}{*}{ SIDS } & 0.99 (0.89 to 1.09) & na & na & 0.99 (0.89 to 1.09$)$ \\
\hline & \multicolumn{4}{|c|}{ OR for days above $50 \mu \mathrm{g} / \mathrm{m}^{3} \mathrm{vs}$. days below $50 \mu \mathrm{g} / \mathrm{m}^{3^{a}}$} \\
\hline Total & 1.10 (0.94 to 1.29$)$ & $0.99(0.78$ to 1.24$)$ & $1.74(1.18 \text { to } 2.58)^{* *}$ & $1.04(0.79$ to 1.37$)$ \\
\hline Cardiorespiratory diseases & 0.80 (0.28 to 2.25$)$ & na & na & $0.93(0.32$ to 2.71$)$ \\
\hline Perinatal circumstances & 1.00 (0.78 to 1.28$)$ & 0.96 (0.72 to 1.29$)$ & 1.36 (0.77 to 2.38$)$ & $0.77(0.34$ to 1.72$)$ \\
\hline $\begin{array}{l}\text { Congenital and } \\
\text { chromosomal abnormalities }\end{array}$ & 1.30 (0.98 to 1.74$)$ & 1.03 (0.69 to 1.54$)$ & $2.32(1.24$ to 4.34$){ }^{* *}$ & 1.38 (0.78 to 2.43$)$ \\
\hline SIDS & 0.94 (0.60 to 1.48$)$ & na & na & $0.88(0.55$ to 1.41$)$ \\
\hline
\end{tabular}

Data are ORs with $95 \%$ Cl. ${ }^{*} P \leq 0.05$ and ${ }^{* *} P \leq 0.01$.

na: not applicable due to low numbers in the specified age class.

${ }^{\text {a }}$ Based on EU limit value. 


\section{Figure legends}

Figure 1. Bidirectional time-stratified case-crossover design. The timeline represents one calendar month. Only control days that were temperature-matched within $2^{\circ} \mathrm{C}$ with the day of event, were selected.

Figure 2. A) Frequency distribution of population-weighted daily mean $\mathrm{PM}_{10}$-concentrations in Flanders (Belgium) during the study period (1998-2006). The arrow indicates the EU limit that may be exceeded up to 35 days per year. B) Spatial distribution of population-weighted daily mean $\mathrm{PM}_{10}$-concentration, expressed as number of days with concentration $>50 \mu \mathrm{g} / \mathrm{m}^{3}$ (map of Belgium; the Flemish Region comprises the area north of the black line excluding the capital region of Brussels in the centre of the country).

Figure 3. Shape of the association between exposure to $\mathrm{PM}_{10}$ and risk of mortality in late neonates, expressed as estimated OR (with $95 \% \mathrm{Cl}$, the grey area), using fractional polynomials and $10 \mu \mathrm{g} / \mathrm{m}^{3}$ as reference; $77 \mu \mathrm{g} / \mathrm{m}^{3}$ is the $99^{\text {th }}$ percentile of exposures during the study period. 


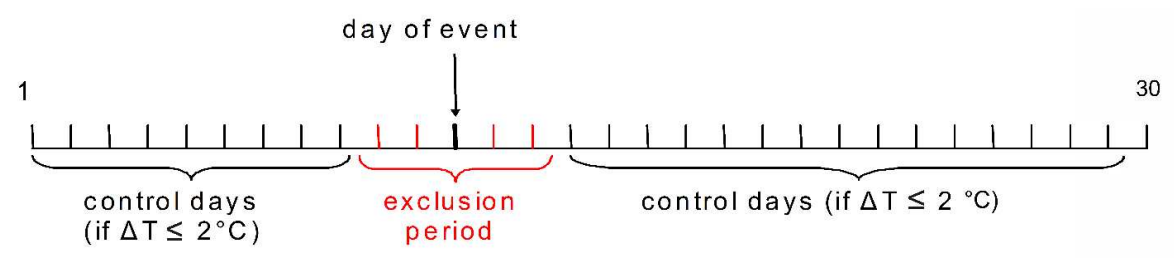

Figure 1. Bidirectional time-stratified case-crossover design. The timeline represents one calendar month. Only control days that were temperature-matched within $2^{\circ} \mathrm{C}$ with the day of event, were selected.

$935 \times 189 \mathrm{~mm}(300 \times 300 \mathrm{DPI})$ 
A

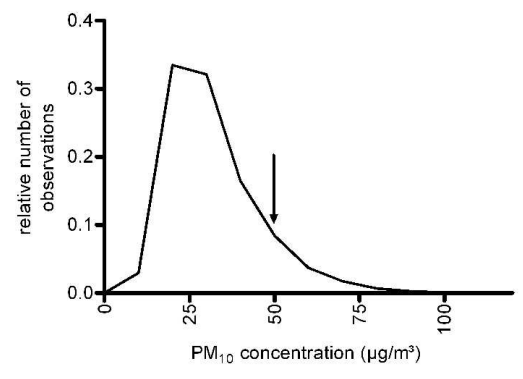

B

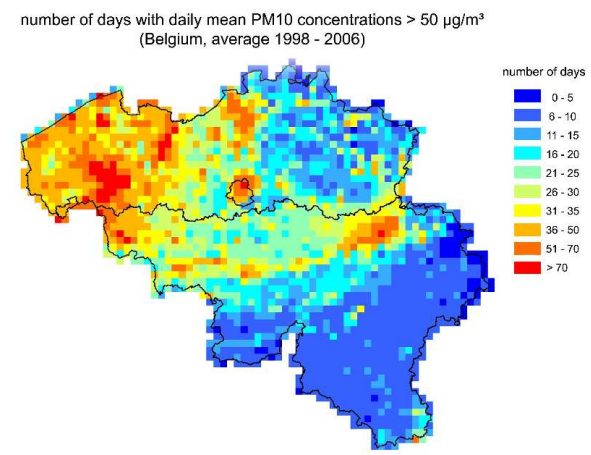

Figure 2. A) Frequency distribution of population-weighted daily mean PM10-concentrations in Flanders (Belgium) during the study period (1998-2006). The arrow indicates the EU limit that may be exceeded up to 35 days per year. B) Spatial distribution of population-weighted daily mean PM10-concentration, expressed as number of days with concentration $>50 \mu \mathrm{g} / \mathrm{m}^{3}$ (map of Belgium; the Flemish Region comprises the area north of the black line excluding the capital region of Brussels in the centre of the country).

$457 \times 198 \mathrm{~mm}(300 \times 300 \mathrm{DPI})$ 


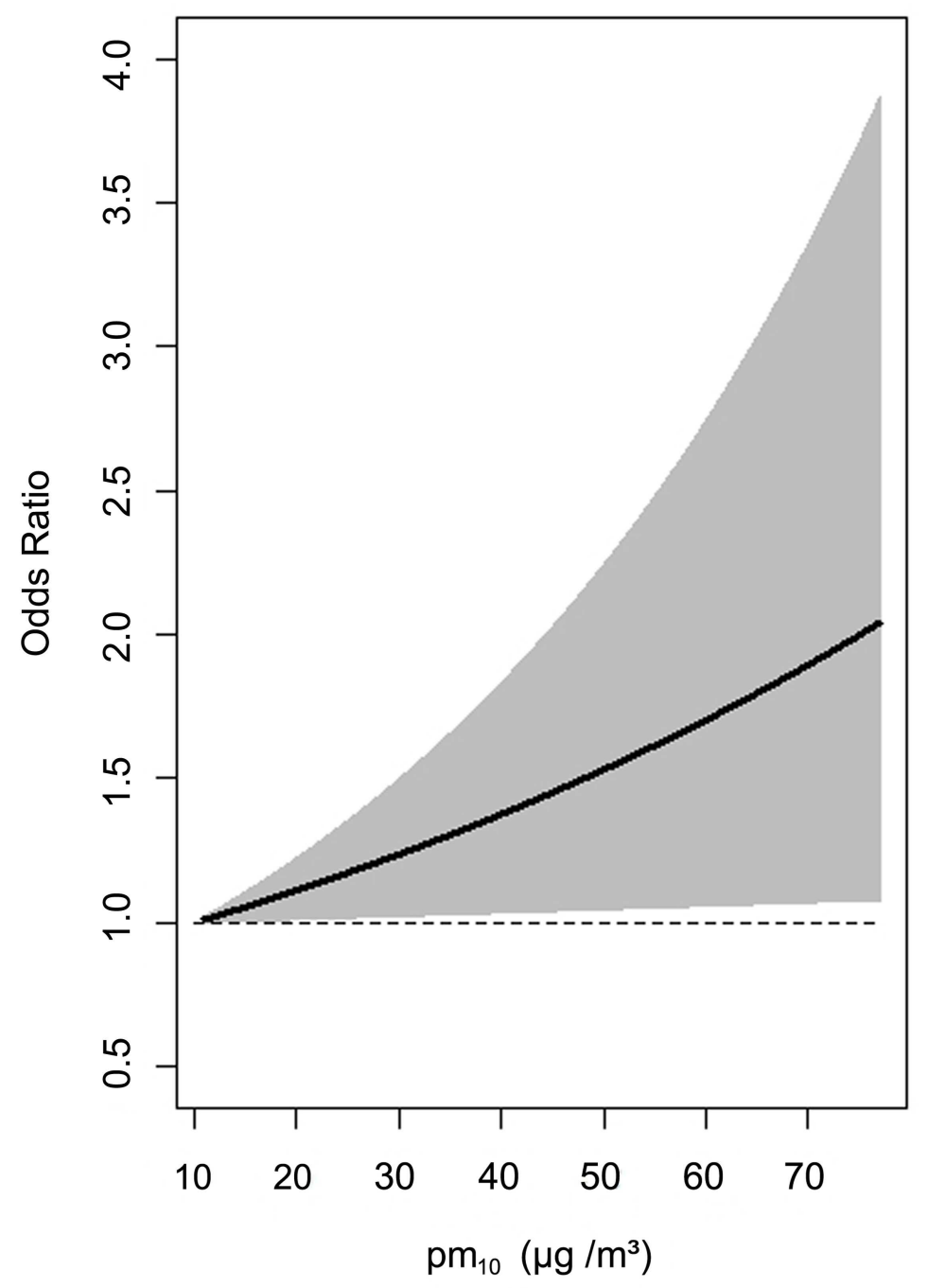

Figure 3. Shape of the association between exposure to PM10 and risk of mortality in late neonates, expressed as estimated OR (with $95 \% \mathrm{CI}$, the grey area), using fractional polynomials and 10 $\mu \mathrm{g} / \mathrm{m}^{3}$ as reference; $77 \mu \mathrm{g} / \mathrm{m}^{3}$ is the 99 th percentile of exposures during the study period. $158 \times 236 \mathrm{~mm}(400 \times 400$ DPI $)$ 